论 文

\title{
非绝热、非周期演化量子相位特征与测量研究
}

\author{
李永放”，马瑞琼，任立庆，王蕾，王兆华，李百宏 \\ 陕西师范大学物理学与信息技术学院，西安 710062 \\ *E-mail: yfli@snnu.edu.cn \\ 收稿日期: 2009-05-15; 接受日期: 2009-09-09 \\ 陕西省自然科学基金资助项目(编号: 2007A04)
}

摘要以二能级原子系统为模型, 细致地研究了非绝热演化量子相位特征. 基于量子 干涉原理，提出了一种测量非绝热演化量子相位的新方法. 且阐述了这种测量方法的物 理机理, 利用这种方法在原子态布居几率的干涉条纹中可直接获得量子相位的分布信息.

关键词

非绝热演化相位

量子干涉效应

二能级原子系统
二能态体系是最简单的量子体系, 同时又是量 子特征最强的体系, 因此在研究光与物质相互作用 时往往会从二能级系统出发. 这样做不仅较容易得 到严格解, 也能够清楚地阐述光与物质相互作用过 程中最重要、最基本的机理. 例如, 近年来人们十分 关注的量子信息提取与处理等问题的研究便是基于 二能态体系而展开, 并取得了丰富的结果. 特别是对 量子力学中的基本物理问题一几何相位的研究显 得尤为活跃. 这是由于与几何相位相关的量子比特 操作有着很强的容错能力 ${ }^{1), 2}$. 几何相位的这一独特 的物理特性为量子计算研究开辟了新的思路 ${ }^{[1-3]}$. 对 几何相位的研究已由早期的绝热、周期、幺正演化的 Berry 相 ${ }^{[4]}$, 逐步推广到非绝热、周期、乡正演化的 $\mathrm{A}-\mathrm{A}$ 相 ${ }^{[5]}$ 和非绝热、非周期、么正演化的一般纯态几 何相位 ${ }^{[6]}$. 近年来的研究又将其推广到么正演化的混 合态几何相位 ${ }^{[7-9]}$ 和非么正演化的混合态几何相位 ${ }^{[10]}$.

然而对几何相位的测量是十分困难的, 尤其是 对非绝热或近似绝热演化几何相位的测量还未见报 道. 而以往的测量几何相位的方法大多是采用核磁
共振或光子回波(统称为回波方法) ${ }^{[11 ~ 17]}$. 本文基于高 斯脉冲作用下的二能级原子模型, 解析描述了非绝 热量子相位特征.

利用量子干涉方法在理论上提出了一个切实可 行的测量非绝热演化量子相位的全新实验方法. 新 的测量方法克服了回波方法的不足之处, 可在布居 几率中直接获得量子相位信息. 这一新方法为研究 探索测量几何相位的新机理和新方法有着重要的科 学意义; 对于了解各种条件下的非绝热演化量子相 位的规律、研究几何相位在量子计算中的应用具有重 要作用.

\section{1 非绝热演化的量子相位}

\section{1 绝热演化情况下一般能级体系的量子相位 ${ }^{[18]}$}

在含时光场作用下, 原子体系会产生瞬时本征 态 $|n(t)\rangle$. 假设 $|n(t)\rangle$ 是含时哈密顿量 $H(t)$ 作用下的 一组力学量完全集的共同本征态, 满足 $H(t)|n(t)\rangle=$

1) Kitaev A Y. Fault tolerant quantum computation with anyons. LANL e-print quant-ph/9707021

2) Preskill J. Fault tolerant quantum computation. LANL e-print quant-ph/9712048

引用格式: 李永放, 马瑞琼, 任立庆, 等. 非绝热、非周期演化量子相位特征与测量研究. 中国科学: 物理学 力学 天文学, 2010, 40: 183 190 
$E_{n}(t)|n(t)\rangle$ 和 $\langle m(t) \mid n(t)\rangle=\delta_{m n}$ 的关系. $E_{n}(t)$ 为瞬 时态 $|n(t)\rangle$ 的能量本征值. 原子体系的波函数表示为 $|\psi(t)\rangle=\sum_{n=1,2} c_{n}(t)|n(t)\rangle$, 且假设量子态波函数满足薛 定谔方程. 这样利用薛定谔方程方程可得到:

$$
\begin{aligned}
\dot{c}_{n}(t)= & -\frac{\mathrm{i}}{\hbar} c_{n}(t) E_{n}(t)-c_{n}(t)\langle n(t) \mid n(t)\rangle \\
& -\sum_{m \neq n} c_{m}(t)\langle n(t) \mid m(t)\rangle,
\end{aligned}
$$

$\langle m(t) \mid n \dot{(t)}\rangle$ 称为绝热参量. 当 $\langle m(t) \mid n \dot{(t)}\rangle=0$ 时称为 严格满足绝热条件; 当 $\langle m(t) \mid n \dot{(t)}\rangle \ll 1$ 称为满足近似 绝热条件. 在严格满足绝热条件下, (1)式的解为

$$
c_{n}(t)=c_{n}(0) \exp \left[-\mathrm{i} \frac{1}{\hbar} \int_{0}^{t} E_{n}\left(t^{\prime}\right) \mathrm{d} t^{\prime}-\int_{0}^{t}\left\langle n\left(t^{\prime}\right) \mid n\left(t^{\prime}\right)\right\rangle \mathrm{d} t^{\prime}\right],
$$

由 $\langle n(t) \mid n(t)\rangle=1$ 可以得到 $\langle n(t) \mid n(t)\rangle$ 为纯虚数. 若 作用光场具有周期演化规律, 由 $\langle n(t) \mid n(t)\rangle$ 导致的相 位表示为 $\gamma_{n}(t)=i \int_{0}^{t}\left\langle n\left(t^{\prime}\right) \mid n \dot{\left(t^{\prime}\right)}\right\rangle \mathrm{d} t^{\prime}$, 称为绝热演化的 几何相位, 即著名的 Berry 相 ${ }^{[4,18,19]}$. 这样(2)式的解 可简化写为

$$
c_{n}(t)=c_{n}(0) \exp \left[\mathrm{i}\left(\Theta_{n}(t)+\gamma_{n}(t)\right)\right],
$$

其中 $\Theta_{n}(t)=-\frac{1}{\hbar} \int_{t_{0}}^{t} E_{n}\left(t^{\prime}\right) \mathrm{d} t^{\prime}$ 称为动力学相, $t_{0}$ 是与原 子系统作用光场的初始作用时刻. 因而原子体系的 波函数可表示为

$$
|\psi(t)\rangle=\sum_{n} c_{n}(0) \exp \left[\mathrm{i} \Theta_{n}(t)\right] \exp \left[i \gamma_{n}(t)\right]|n(t)\rangle .
$$

\section{2 二能级体系中非绝热与近似绝热演化的量子 相位}

当体系近似满足或不满足绝热条件时, 便会存 在透热过程. 这时令 $c_{n}(t)=\lambda_{n}(t) \exp \left[\mathrm{i}\left(f_{n}(t)+\chi_{n}(t)\right]\right.$, 其中 $f_{n}(t)=\Theta_{n}(t)+\gamma_{n}(t)$. 在后面可以看到引入的相 位因子 $\chi_{n}(t)$ 是与系统的透热过程或非绝热过程相 关. 将所令的波函数解代入(1)式得到:

$$
\begin{aligned}
& \dot{\lambda}_{n}(t)+\mathrm{i} \lambda_{n}(t) \dot{\chi}_{n}(t) \\
& =-\sum_{m \neq n} \lambda_{m}(t) \exp \left[\mathrm{i}\left(\left(f_{m}(t)+\chi_{m}(t)\right)-\left(f_{n}(t)+\chi_{n}(t)\right)\right)\right] \\
& \quad \times\langle n(t) \mid m(t)\rangle .
\end{aligned}
$$

为了简化上式, 令 $\left|n_{f}(t)\right\rangle=\exp \left[\mathrm{i} f_{n}(t)\right]|n(t)\rangle$, 并考 虑简单的二能级系统情况 $(n, m=1,2)$. 同时假设 $\langle 1(t) \mid 2(t)\rangle=\mu(t) \exp [\mathrm{i} \beta(t)]$ (根据共轭关系有: $\langle 2(t) \mid \dot{1}(t)\rangle=\mu(t) \exp [-\mathrm{i} \beta(t)])$, 其中 $\mu(t), \beta(t)$ 均为实 数, 且 $\mu(t)$ 称为透热振幅, $\beta(t)$ 称为透热相位. 它们 表征着能态 $|n(t)\rangle$ 间的能量交换关系. 这样对于二能 级系统(5)式可以简化为

$$
\left\{\begin{array}{l}
\mathrm{i} \lambda_{1}(t) \dot{\chi_{1}}(t)+\dot{\lambda_{1}}(t) \\
=-\lambda_{2}(t) \mu(t) \exp \left[\mathrm{i}\left(\chi_{2}(t)-\chi_{1}(t)+\beta(t)\right)\right], \\
-\mathrm{i} \lambda_{2}(t) \dot{\chi_{2}}(t)+\dot{\lambda_{2}}(t) \\
=\lambda_{1}(t) \mu(t) \exp \left[\mathrm{i}\left(\chi_{2}(t)-\chi_{1}(t)+\beta(t)\right)\right] .
\end{array}\right.
$$

比较上述两式的虚部与实部可分别得到一组方程:

$$
\left\{\begin{array}{l}
\dot{\lambda_{1}}(t)=-\lambda_{2}(t) \mu(t) \cos \left[\chi_{2}(t)-\chi_{1}(t)+\beta(t)\right], \\
\dot{\lambda_{2}}(t)=\lambda_{1}(t) \mu(t) \cos \left[\chi_{2}(t)-\chi_{1}(t)+\beta(t)\right],
\end{array}\right.
$$

$\left\{\begin{array}{l}\lambda_{1}(t) \dot{\chi_{1}}(t)=-\lambda_{2}(t) \mu(t) \sin \left[\chi_{2}(t)-\chi_{1}(t)+\beta(t)\right], \\ \lambda_{2}(t) \dot{\chi_{2}}(t)=-\lambda_{1}(t) \mu(t) \sin \left[\chi_{2}(t)-\chi_{1}(t)+\beta(t)\right] .\end{array}\right.$

由(7)式的两方程组可得到:

$$
\begin{aligned}
& \frac{\mathrm{d}}{\mathrm{d} t}\left(\lambda_{1}(t)^{2}+\lambda_{2}(t)^{2}\right)=0, \\
& \dot{\chi_{1}}(t) \lambda_{1}(t)^{2}=\lambda_{2}(t)^{2} \dot{\chi_{2}}(t), \\
& \dot{\lambda_{2}}(t)-\dot{\lambda_{1}}(t)=\left(\lambda_{1}(t)+\lambda_{2}(t)\right) \mu(t) \\
& x \cos \left[\chi_{2}(t)-\chi_{1}(t)+\beta(t)\right], \\
& \dot{\chi_{2}}(t)-\dot{\chi_{1}}(t)=\left(\frac{\lambda_{2}(t)}{\lambda_{1}(t)}-\frac{\lambda_{1}(t)}{\lambda_{2}(t)}\right) \mu(t) \\
& \times \sin \left[\chi_{2}(t)-\chi_{1}(t)+\beta(t)\right] .
\end{aligned}
$$

(8a)式中表明 $|n(t)\rangle$ 态几率振幅间的守恒关系; $(8 b)$ 式 表明相位 $\chi_{1,2}(t)$ 与对应能态 $|n(t)\rangle$ 上布居几率的伴随 关系. 根据(7a)式得到: 


$$
\chi_{2}(t)-\chi_{1}(t)=\arccos \left[\frac{\dot{\lambda}_{2}(t)}{\lambda_{1}(t) \mu(t)}\right]-\beta(t) .
$$

上式表明非绝热演化引起的相位 $\chi_{2}(t)-\chi_{1}(t)+\beta(t)$ 是由 $\lambda_{1}(t), \lambda_{2}(t)$ 以及 $\mu(t)$ 决定的. 一般情况下 (7) 和 (8) 式很难求解, 但当考虑体系满足近似绝热的特殊 情况下, 可获得相位 $\chi_{2}(t)-\chi_{1}(t)$ 的解析表示.

由(7a)式可以看到, 当体系满足近似绝热条件 $\mu(t)=|\langle 1(t) \mid 2(t)\rangle| \ll 1$ 时, 瞬时量子态的布居几率波 函数的振幅 $\lambda_{1}(t), \lambda_{2}(t)$ 成为与时间无关的常量 $\lambda_{1}, \lambda_{2}$. 这样 $(8 \mathrm{c})$ 式中等式左边 $\dot{\lambda}_{2}(t)-\dot{\lambda}_{1}(t)=0$, 因为 $\mu(t)$ 很 小但满足关系 $\mu(t) \neq 0$, 且不论 $\lambda_{1}(t)+\lambda_{2}(t)$ 是否为零 均有 $\cos \left[\chi_{2}(t)-\chi_{1}(t)+\beta(t)\right]=0$, 所以得到 $\chi_{2}(t)-$ $\chi_{1}(t)+\beta(t)=(4 k \pm 1) \frac{\pi}{2}$. 将其代入 $(8 \mathrm{~d})$ 和 $(7 \mathrm{~b})$ 式并积 分可得

$$
\begin{aligned}
\chi_{2}(t)-\chi_{1}(t) & =\left(\frac{\lambda_{2}^{2}-\lambda_{1}^{2}}{\lambda_{1} \lambda_{2}}\right) \int_{t_{0}}^{t} \mu(t) \mathrm{d} t+\chi_{2}\left(t_{0}\right)-\chi_{1}\left(t_{0}\right), \\
& \left\{\begin{array}{l}
\chi_{2}(t)=\chi_{2}\left(t_{0}\right)-\frac{\lambda_{1}}{\lambda_{2}} \int_{t_{0}}^{t} \mu(t) \mathrm{d} t, \\
\chi_{1}(t)=\chi_{1}\left(t_{0}\right)-\frac{\lambda_{2}}{\lambda_{1}} \int_{t_{0}}^{t} \mu(t) \mathrm{d} t
\end{array}\right.
\end{aligned}
$$

比较 $(10)$ 式和 $\chi_{2}(t)-\chi_{1}(t)+\beta(t)=(4 k \pm 1) \frac{\pi}{2}$, 可以得 到:

$$
\begin{aligned}
& \beta(t)=\left(\frac{\lambda_{1}^{2}-\lambda_{2}^{2}}{\lambda_{1} \lambda_{2}}\right) \int_{t_{0}}^{t} \mu\left(t^{\prime}\right) \mathrm{d} t^{\prime}, \\
& \chi_{2}\left(t_{0}\right)-\chi_{1}\left(t_{0}\right)=(4 k \pm 1) \frac{\pi}{2} .
\end{aligned}
$$

从(11)式的两个结果中可以看到, $\chi_{1,2}(t)$ 是由初始相 位和对透热振幅 $\mu(t)$ 的积分两部分组成. 对透热振 幅 $\mu(t)$ 的积分就是透热过程对相位的累积过程, 因 而称 $\chi_{1,2}(t)$ 为非绝热演化相位. 而两个能态所对应非 绝热演化的初始相位差则是一个确定值, 它的存在 是由于在外光场作用下, 能级的 Stark 分裂过程所导 致的结果. 它与具体作用于二能级系统的光场初始 状态有关. 而且在后面研究高斯光场作用时可以看
到, 非绝热演化相位 $\chi_{1,2}(t)$ 仅仅是状态的函数, 与过 程和周期演化无关，是一个非绝热、非周期演化的相 位. 另外引入的透热相位 $\beta(t)$ 恰好反映了两个 Stark 能级间的透热关系. 因此说(9)式是非绝热演化相位 的一般表示, 而(10)和(11)式则是在近似绝热条件下本 文所获得的重要结果. 对于每个 Stark 分裂能级(或缀 饰态), 它的非绝热演化的初始相位很难确定, 但具 有确定值的两个非绝热演化相位差则是可以测量的.

\section{3 高斯脉冲光场作用下的二能级体系非绝热演 化量子相位}

考虑强高斯脉冲光场 $E_{1}(t)=\varepsilon(t) \exp \left(\mathrm{i} \omega_{L 1} t\right)+$ c.c. 作用下由态 $|g\rangle$ 和激发态 $|e\rangle$ 组成的二能级体系, 光 场的振幅部分为高斯脉冲 $\varepsilon(t)=\varepsilon_{10} \exp \left[-\left(t / \tau_{1}\right)^{2}\right], \varepsilon_{10}$ 为脉冲振幅, $\tau_{1}$ 为脉冲宽度; $\omega_{L 1}$ 为激光场的频率. 在旋转坐标系下体系的哈密顿量写为

$$
H(t)=\frac{1}{2} \hbar\left(\begin{array}{cc}
\Delta_{1} & \Omega_{1}^{*}(t) \\
\Omega_{1}(t) & -\Delta_{1}
\end{array}\right),
$$

其中 $\Delta_{1}=\omega_{e g}-\omega_{L 1}$ 是强驱动场与原子系统作用时的 失谐量, $\omega_{e g}$ 是基态与激发态间的频率差; $\Omega_{1}(t)=$ $-\mu_{e g} \varepsilon(t) / \hbar\left(\Omega_{10}=-\mu_{e g} \varepsilon_{10} / \hbar\right)$ 为 Rabi 频率; $\mu_{e g}$ 是基 态与激发态间偶极跃迁矩阵元. 原子能态将在外场 作用下发生移动(Stark 分裂), 形成新的瞬时本征态. 它们分别表示为: $\left|\phi_{+}(t)\right\rangle=\sin \alpha(t)|g\rangle+\cos \alpha(t)|e\rangle$, $\left|\phi_{-}(t)\right\rangle=\cos \alpha(t)|g\rangle-\sin \alpha(t)|e\rangle$, 其中 $\alpha(t)$ 定义为: $\tan 2 \alpha=\frac{\Omega_{1}(t)}{\Delta_{1}}$. 与本征矢对应的本征值为 $\hbar \varepsilon_{ \pm}(t)=$ $\pm \frac{\hbar}{2} \sqrt{\Delta_{1}^{2}+\left|\Omega_{1}(t)\right|^{2}}= \pm \frac{\hbar}{2} \Omega_{\mathrm{eff}}(t), \quad \Omega_{\mathrm{eff}}(t)=\sqrt{\Delta_{1}^{2}+\left|\Omega_{1}(t)\right|^{2}}$ 称为有效拉比频率. 根据 $\left|\phi_{ \pm}(t)\right\rangle$ 的表示以及

$$
\begin{aligned}
\tan 2 \alpha(t)=\frac{\Omega_{1}(t)}{\Delta_{1}} \text { 可得 } & \\
\left\langle\phi_{-}(t) \mid \phi_{+}(t)\right\rangle & =-\left\langle\phi_{+}(t) \mid \phi_{-}(t)\right\rangle \\
& =\dot{\alpha(t)}=\frac{\dot{\Omega_{1}(t) \Delta_{1}}}{2\left(\varepsilon_{+}(t)-\varepsilon_{-}(t)\right)^{2}} .
\end{aligned}
$$

根据前面的讨论以 $\left|\phi_{+}(t)\right\rangle$ 替换 $|2(t)\rangle,\left|\phi_{-}(t)\right\rangle$ 对 应 $|1(t)\rangle$. 同样以 $\chi_{+}(t)$ 替换 $\chi_{2}(t), \chi_{-}(t)$ 替换 $\chi_{1}(t)$. 
结合定义 $\mu(t)=|\langle 1(t) \mid 2 \dot{(t)}\rangle|$ 可以得到 $\int_{t_{0}}^{t} \mu\left(t^{\prime}\right) \mathrm{d} t^{\prime}=$ $\int_{t_{0}}^{t} \dot{\alpha}\left(t^{\prime}\right) \mathrm{d} t^{\prime}=\alpha(t)-\alpha\left(t_{0}\right)$, 其中 $t_{0}$ 是高斯脉冲的起始时 刻. 这样从(10)和(11)式可知, 此时非绝热相 $\chi_{+}(t)$ $\left(\chi_{-}(t)\right)$ 与过程无关, 仅与初始时刻和观测时刻的 $\alpha(t)$ 有关. 因此 $\chi_{ \pm}(t)$ 是一个非绝热、非周期演化的 相位. 此时非绝热演化相(10)式可以表示为

$$
\begin{aligned}
\chi_{+}(t)-\chi_{-}(t)= & \left(\frac{\lambda_{+}\left(t_{0}\right)^{2}-\lambda_{-}\left(t_{0}\right)^{2}}{\lambda_{-}\left(t_{0}\right) \lambda_{+}\left(t_{0}\right)}\right)\left(\alpha(t)-\alpha\left(t_{0}\right)\right) \\
& +(4 k \pm 1) \frac{\pi}{2} .
\end{aligned}
$$

在高斯脉冲与二能级系统非共振作用 $\Delta_{1} \neq 0$ 的 一般情况, 且同时体系满足近似绝热条件时, 则有 $\lambda_{-}\left(t_{0}\right)=\cos \alpha\left(t_{0}\right), \lambda_{+}\left(t_{0}\right)=\sin \alpha\left(t_{0}\right)$, 再根据 $\tan 2 \alpha=$ $\frac{\Omega_{1}(t)}{\Delta_{1}}$ 可以得到 $\alpha(t)=\frac{1}{2} \arctan \left(\frac{\Omega_{1}(t)}{\Delta_{1}}\right)$. 这样由(16) 式所表示的非绝热演化相位差便可表示为

$$
\chi_{-}(t)-\chi_{+}(t)=\frac{\Delta_{1}}{\Omega_{1}\left(t_{0}\right)} \arctan \left[\frac{\Omega_{1}(t)}{\Delta_{1}}\right]+(4 k \pm 1) \frac{\pi}{2} .
$$

(17)式表明失谐量越大非绝热演化相位差就越大, 如 图 1 所示. 图 1 中计算时取(17)式最后以项为 $-\pi / 2$. 在近似绝热条件下, 非绝热演化量子相位差随时间 和作用光场失谐量的变化都是对称分布的.

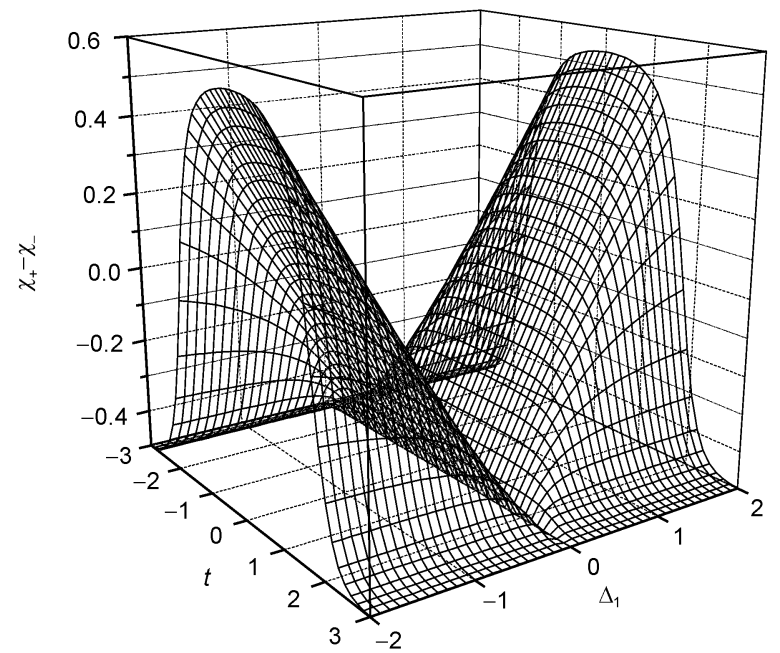

图 1 非绝热演化相位差随脉冲作用时间和失谐量的变化 关系
由于高斯光场在时域的对称性, 导致能级分裂 的对称性, 满足 $\Omega_{\mathrm{eff}}\left(-t_{1}\right)=\Omega_{\mathrm{eff}}\left(t_{1}\right)$, 如图 2 所示. 所以 由周期复归而引入了 Berry 几何相 ${ }^{[4,19,20]}$, 这样系统 的波函数可表示为

$$
\begin{aligned}
|\psi(t)\rangle= & \sin \alpha\left(t_{0}\right) \exp \left[\mathrm{i} \chi_{+}(t)\right] \\
& \times \exp \left[-\mathrm{i}\left(\frac{1}{2} \theta(t)-\gamma_{+}(t)\right)\right]\left|\phi_{+}(t)\right\rangle \\
& +\cos \alpha\left(t_{0}\right) \exp \left[\mathrm{i} \chi_{-}(t)\right] \\
& \times \exp \left[\mathrm{i}\left(\frac{1}{2} \theta(t)+\gamma_{-}(t)\right)\right]\left|\phi_{-}(t)\right\rangle,
\end{aligned}
$$

其中 $\theta(t)=\int_{t_{0}}^{t} \sqrt{\Delta^{2}+\left|\Omega_{1}\left(t^{\prime}\right)\right|^{2}} \mathrm{~d} t^{\prime}$ 为有效脉冲面积, $\gamma(t)=$ $\pi(1-\cos 2 \alpha(t))$.

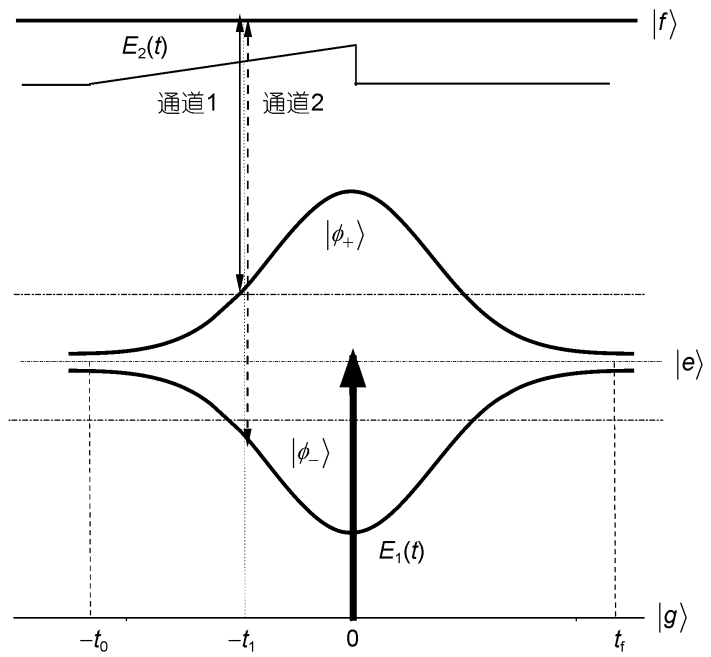

图 2 高斯脉冲光场作用下的能级分裂和三角波的探测 过程示意图

在文献[19,20]报道中, 我们提出的在同一能态中 获得的双通道量子干涉效应，实现了对周期演化的 Berry 几何相位的测量. 同样, 利用非对称的三角波 光场探测量子态，在不同能态间实现量子干涉效应， 可实现对非绝热、非周期演化量子相位的测量, 并利 用密度矩阵方程数值解验证了这一方法的正确性.

\section{2 弱探测场作用下系统终态 $|f\rangle$ 粒子布居的 演化}

在通常情况下(11)式中表示的非绝热演化相位 
中初始相位很难确定, 也是很难探测的. 针对(13)和 (17)表示的非绝热演化相位差, 我们设计了一个实验 方案, 实现了对非绝热演化相位差的测量. 其方法是: 利用一个三角波弱探测场, 它的作用时间仅仅是制 备量子态的强高斯脉冲光场的一半, 探测 Stark 能态 的前半个演化周期, 没有对能态的另外对称周期点 测量. 因而探测过程与能态的周期演化无关, 从而无 法观测其中的 Berry 几何相位. 采用三角波探测时使 用三角波探测的作用就是为了剪裁掉高斯脉冲后沿 的跃迁对结果的影响, 从而避免了探测过程中 Berry 几何相的影响. 只有当探测场的与作用于原子的强 场时间宽度一致时方能实现对一对对称周期演化的 测量 ${ }^{[19 \sim 21]}$.

当探测光场的频率带宽较宽, 可以在同一瞬时 同时激发两个 Stark 能级(或缀饰态)到终态 $|f\rangle$ 的跃 迁, 如图 2 所示. 这样便可同时完成双通道的激发过 程而产生量子干涉效应. 通过观测终态到其他能级 的跃迁或辐射, 便可测量到反映量子干涉结果的荧 光信号. 其苂光信号中便包含了被测量子态的量子 相位信息.

根据上面所述的测量机理, 由(18)式, 激发态 $|e\rangle$ 波函数可以表示为

$$
\begin{aligned}
C_{e}(t) & =\langle e \mid \psi(t)\rangle \\
& =\lambda_{+}\left(t_{0}\right) \cos \alpha(t) \exp \left[\mathrm{i}\left(\frac{1}{2} \theta(t)+\gamma_{+}(t)+\chi_{+}(t)\right)\right] \\
& -\lambda_{-}\left(t_{0}\right) \sin \alpha(t) \exp \left[\mathrm{i}\left(-\frac{1}{2} \theta(t)+\gamma_{-}(t)+\chi_{-}(t)\right)\right] \\
& =C_{+}(t)+C_{-}(t),
\end{aligned}
$$

其 中 $\chi_{-}(t)-\chi_{+}(t)=\frac{\Delta_{1}}{\Omega_{1}\left(t_{0}\right)} \arctan \left[\frac{\Omega_{1}(t)}{\Delta_{1}}\right]+(4 k+1) \frac{\pi}{2}$. 由于是弱场探测过程, 因此利用微扰理论和薛定谔 方程, 从量子态 $\left|\phi_{ \pm}(t)\right\rangle$ 跃迁到终态 $|f\rangle$ 的布居几率振 幅的表示写为

$$
\begin{aligned}
C_{f \pm} & =\left\langle f\left|-\mu \cdot E_{2}(t)\right| \phi_{ \pm}(t)\right\rangle \\
& =\frac{\mathrm{i}}{2} \int_{t_{s}}^{t} \Omega_{2}\left(t^{\prime}\right) \exp \left[ \pm \mathrm{i}\left|\Delta_{2}\right| t^{\prime}\right] C_{ \pm}\left(t^{\prime}\right) \mathrm{d} t^{\prime},
\end{aligned}
$$

其中 $\Delta_{2}=\omega_{L 2}-\omega_{f e}$ 为探测场的失谐量, 由图 2 可以看 出, 对于任意的 $\left|\Delta_{2}\right|<\frac{1}{2}\left|\Omega_{1}(0)\right|\left(\left|\Omega_{1}(0)\right|\right.$ 是瞬时能态位
移最大值), 都将共振激发量子态的跃迁. 从瞬时态 的角度考察, $\left|\Delta_{2}\right|$ 则是不同时刻共振激发的探测场频 率. 考虑到探测场作用非常弱, 可以忽略非共振跃迁 的影响. 这样在不同时刻瞬时态对探测场的响应便 可以视为彼此独立的, 因此可根据驻相法近似处理 (20)式的积分 ${ }^{[18,19]}$, 将其简化为共振时刻的瞬态响 应. 满足驻相法近似时, 共振时刻 $t$ 与 $\Delta_{2}$ 间的对应关 系为: $t= \pm \tau_{1} \sqrt{-\frac{1}{2} l_{n}\left[4\left|\Delta_{2}^{2}-\Delta_{1}^{2}\right| / \Omega_{10}^{2}\right]}$. 另外由于系统 的总相移是各态相移按权重叠加的结果 ${ }^{[17]}$, 因而 弱场探测到的则是被测量子态受权重因子影响下的 结果.

根据前面所述的探测原理, 两个量子态向终态 跃迁的几率是相干叠加:

$$
\begin{aligned}
\left|C_{\mathrm{I}}(t)\right|^{2}= & \left|C_{f+}(t)+C_{f-}(t)\right|^{2} \\
= & \frac{1}{4} \Omega_{2}^{2}(t)\left(\left|\lambda_{+}\left(t_{0}\right)\right|^{2} \cos ^{2} \alpha(t)+\left|\lambda_{-}\left(t_{0}\right)\right|^{2} \sin ^{2} \alpha(t)\right. \\
& \left.-2 \lambda_{+}\left(t_{0}\right) \lambda_{-}\left(t_{0}\right) \cos \alpha(t) \sin \alpha(t) \cos \varphi(t)\right), \quad(21)
\end{aligned}
$$

其中

$$
\begin{aligned}
\varphi(t)= & \theta(t)+\left(\left|\lambda_{+}\left(t_{0}\right)\right|^{2} \gamma_{+}(t)-\left|\lambda_{-}\left(t_{0}\right)\right|^{2} \gamma_{-}(t)\right) \\
& +\left(\left|\lambda_{+}\left(t_{0}\right)\right|^{2} \chi_{+}(t)-\left|\lambda_{-}\left(t_{0}\right)\right|^{2} \chi_{-}(t)\right)+2|\Delta| t,
\end{aligned}
$$

非绝热相位差因子为

$$
\begin{aligned}
& \left|\lambda_{+}\left(t_{0}\right)\right|^{2} \chi_{+}(t)-\left|\lambda_{-}\left(t_{0}\right)\right|^{2} \chi_{-}(t) \\
& =\left(\left|\lambda_{+}\left(t_{0}\right)\right|^{2} \frac{\lambda_{+}\left(t_{0}\right)}{\lambda_{-}\left(t_{0}\right)}-\left|\lambda_{-}\left(t_{0}\right)\right|^{2} \frac{\lambda_{-}\left(t_{0}\right)}{\lambda_{+}\left(t_{0}\right)}\right) \alpha(t) \\
& \quad-\left(\left|\lambda_{+}\left(t_{0}\right)\right|^{2}-\left|\lambda_{-}\left(t_{0}\right)\right|^{2}\right) \frac{\pi}{2} .
\end{aligned}
$$

由于探测过程与能态的周期演化无关, 因此无法观测 其中的 Berry 几何相位, 这样 $\gamma_{+}(t), \gamma_{-}(t)$ 因子的影响 均值为零, 可以忽略其作用. 由此(22)式可以简化为

$$
\begin{aligned}
\varphi(t)= & \theta(t)+\left(\left|\lambda_{+}\left(t_{0}\right)\right|^{2} \chi_{+}(t)-\left|\lambda_{-}\left(t_{0}\right)\right|^{2} \chi_{-}(t)\right) \\
& +2\left|\Delta_{2}\right| t,
\end{aligned}
$$

其中第一项反映的是驱动场制备瞬时态 $\left|\phi_{+}\right\rangle$和 $\left|\phi_{-}\right\rangle$ 的动力学相相位差. 第二项源自不同瞬时态的量子 相位对干涉的贡献, 是与瞬时态的状态波函数有关 的量子干涉项. 前两项与量子相位相关, 主导着量子 干涉. 最后一项代表了不同频率的探测场间的光学 
干涉作用.

\section{1 非共振作用情况下的非绝热演化相位的测量}

考虑高斯脉冲与二能级系统离共振作用时, 只 要满足近似绝热演化条件, 便可根据(21)式获得终态 $|f\rangle$ 粒子布居的干涉图. 图 3(a)反映了量子干涉对终 态布居数的影响, 其中实线是利用密度矩阵方程 $\mathrm{i} \hbar \frac{\partial}{\partial t} \rho=\left(H_{I}, \rho\right)$ 得到的数值解模拟结果. 由于密度矩 阵方程的变量中不显含量子相位因子, 因此利用密 度矩阵方法求数值解是对方程 (21) 解析结果的很好 验证, 虚线是 (21) 式解析解的模拟结果. 比较可见, 除了在 $\Delta_{2}$ 等于零点处之外, 其余部分的振荡周期和 峰值位置吻合较好. 而在 $\Delta_{2}$ 等于零点, 由于在这个 区间绝热条件不符合而导致了和数值解结果不相符. 这说明(21)式中的相位因子准确地描述体系中量子 干涉对终态布居数的影响. 另外高斯脉冲与二能级 系统离共振作用时, 两量子态权重不相等, 因而参与 干涉的两几率波不是等振幅干涉.

在一般情况下, 描述体系的单态演化时可以将 微弱的透热演化所累积的相位忽略, 但是如果量子 干涉是由于两个量子态之间发生时, 微弱的透热所
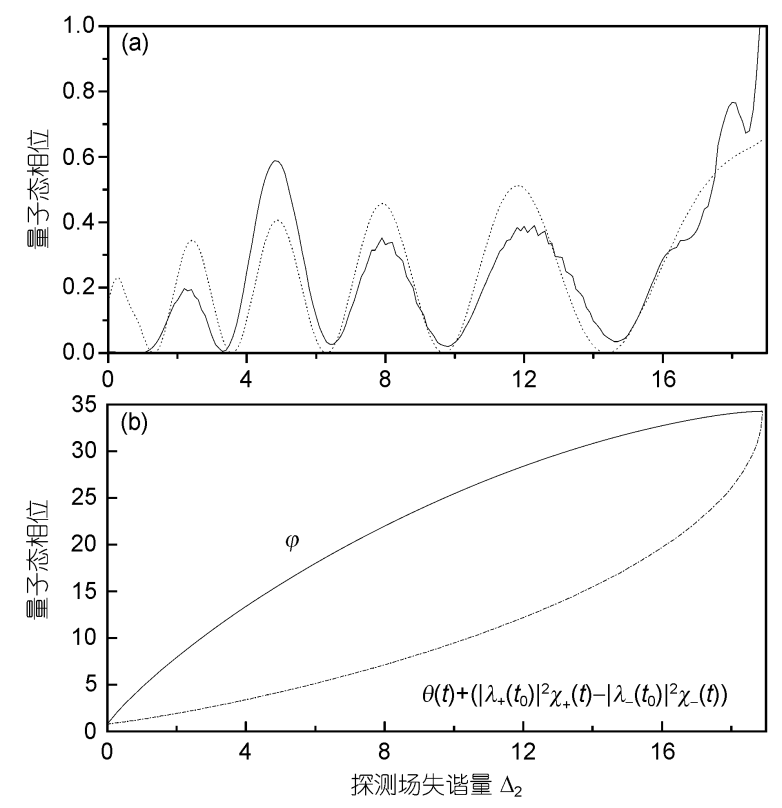

图 3

(a) 终态粒子布居的干涉图, 虚线为(21)式解析解的计算结果, 实 线为密度矩阵方程的数值解结果; (b) 量子相位分布, 实线为总相位 分布, 虚线为量子相位分布; 参数: $\Omega_{10}=37.8, \Omega_{20}=1.77, \tau_{1}=1, \Delta_{1}=1$
引入的相差的影响便不可忽略了. 显然在频域内可 以利用量子干涉提取出非绝热演化相位差, 并且这 个相差因子与两量子态的权重因子有关, 且不依赖 于周期演化.

由于弱场探测时满足与所制备的量子态点点共 振(驻相法要求), 因此量子相位随 $\Delta_{2}$ 的变化就反映 了所制备量子态的量子相位的分布情况, 如图 3(b)所 示. 图 3(b)中实线表示量子干涉的总相位; 虚线则是 量子态的量子相位随 $\Delta_{2}$ 的演化关系, 它反映了体系 的量子相位在频域的分布状况. 而两个曲线之差则 是反映探测光场对量子相位的影响, 可见探测过程 的影响是不容忽视的.

\section{2 量子干涉效应与非绝热演化几何相位的测量}

在高斯脉冲与二能级系统共振作用时, 在高斯 脉冲的起始时刻, 瞬时能态处于简并状态, 因此存在 两个透热点, 但仍满足近似绝热条件. 这时可有 $\alpha(t)=\frac{\pi}{4}, \cos \alpha(t)=\sin \alpha(t)=1 / \sqrt{2}$ 和 $\lambda_{+}(t)=\lambda_{-}(t)=$ $\frac{1}{\sqrt{2}}$. 由 (16) 式可以得到: $\chi_{+}(t)-\chi_{-}(t)=(4 k \pm 1) \frac{\pi}{2}$. 这样(21)式简化为

$$
|C(t)|^{2}=\frac{1}{4} \Omega_{2}^{2} \sin ^{2}\left[\frac{\varphi(t)}{2}\right],
$$

其中 $\varphi(t)=2\left|\Delta_{2}\right| t+\theta(t)+\frac{1}{2}\left(\chi_{+}\left(t_{0}\right)-\chi_{-}\left(t_{0}\right)\right)$. 同样由 于不是对一个周期演化的探测过程, 因此 Berry 相位 则无法观测, 而忽略其影响. 将 $\chi_{+}(t)-\chi_{-}(t)=$ $(4 k \pm 1) \frac{\pi}{2}$ 带入, 取其中为减号, 其相位简化为

$$
\varphi(t)=2\left|\Delta_{2}\right| t+\theta(t)-\frac{1}{4} \pi+2 k \pi .
$$

图 4 是利用密度矩阵方程数值模拟的终态布居 结果. 根据公式 (24)和 (25) 可知, 当 $\varphi(t)=2 n \pi$ 时, 粒 子布居几率为最小值; 当 $\varphi(t)=(2 n+1) \pi$ 满足最大值 条件. 且已知 $\theta(t)$ 的大小时, 便可由图 4 确定与布居 分布的最大和最小值对应的 $\Delta_{2}$. 再利用驻相法近似 得到共振时刻 $t$ 与 $\Delta_{2}$ 间的对应关系: $t=$ $\pm \tau_{1} \sqrt{-l_{n}\left[2\left|\Delta_{2}\right| / \Omega_{10}\right]}$, 我们便可获得非绝热演化相位差 $\frac{1}{2}\left(\chi_{+}\left(t_{0}\right)-\chi_{-}\left(t_{0}\right)\right)$ 的大小, 即实现对非绝热演化相 位的测量. 表 1 便是利用图 4 的布居几率分布获得的 


\section{表 1 非绝热演化相位的测量结果}

\begin{tabular}{|c|c|c|c|c|c|c|c|c|}
\hline \multirow[b]{2}{*}{$\Delta_{2}$} & \multicolumn{4}{|c|}{ 峰值 $\varphi=(2 n+1) \pi$} & \multicolumn{4}{|c|}{ 谷值 $\varphi=2 n \pi$} \\
\hline & 2.47 & 4.93 & 7.91 & 11.68 & 3.61 & 6.34 & 9.66 & 14.13 \\
\hline$\varphi(\Delta) / \pi$ & 2.72 & 4.75 & 6.75 & 8.75 & 3.71 & 5.75 & 7.75 & 9.75 \\
\hline$n$ & 1 & 2 & 3 & 4 & 2 & 3 & 4 & 5 \\
\hline$\Delta \chi_{ \pm} / \pi$ & 0.28 & 0.25 & 0.25 & 0.25 & 0.29 & 0.25 & 0.25 & 0.25 \\
\hline
\end{tabular}

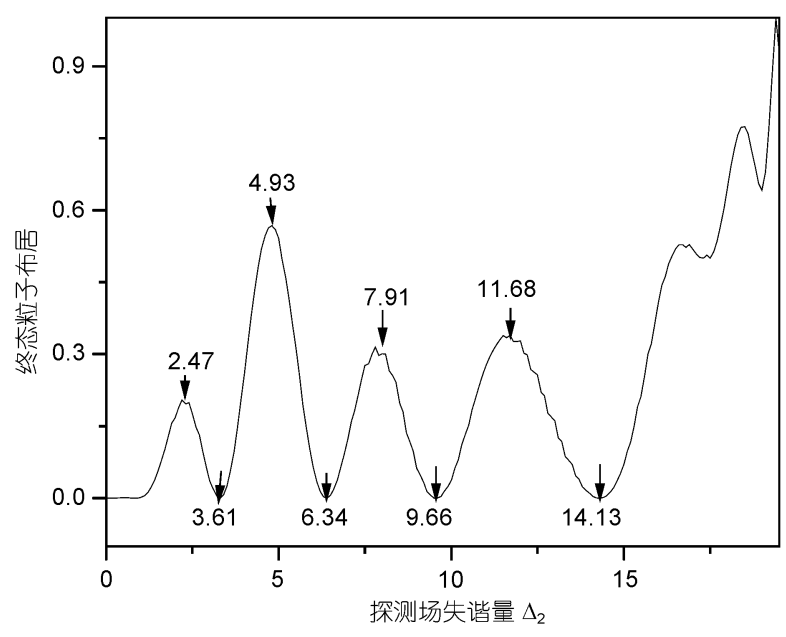

图 4 终态布居数的密度矩阵方程数值解的模拟结果 计算参数 $\Omega_{10}=37.8, \Omega_{20}=1.77, \tau_{1}=1, \Delta_{1}=0$
测量结果. 其中除了 $\Delta_{2}$ 接近零点附近之外, 其余的结果 显示出 $\Delta \chi_{ \pm}=\frac{1}{2}\left(\frac{\pi}{2}\right)$. 前面的 $\frac{1}{2}$ 来至于瞬时能态的权重 因子, 而 $\pi / 2$ 则是由于非绝热演化所导致的初始相位差.

\section{3 结论}

文中详细地分析了在高斯光场作用下所制备的 瞬时能态间由于透热过程所引发的量子相位; 结果 表明其相位特征与过程无关仅与透热的状态相关. 经过密度矩阵方程数值解的验正, 文中所提出的测 量非绝热演化相位的新方法是正确的, 这为量子态 和量子相位的测量研究提供了新的思路, 对于量子 测量、量子计算等方面的理论与实验研究具有重要的 参考价值.

\section{参考文献}

1 Jonathan J A, Vedral V, Ekert A, et al. Geometric quantum computation with NMR. Nature, 2000, 403: 869-871[DOI]

2 Leibfried D, DeMarco B, Meyer V, et al. Experimental demonstration of a robust, high-fidelity geometric two ion-qubit phase gate. Nature, 2003, 422: 412-415[DOI]

3 Zhu S L, Wang Z D. Implementation of universal quantum gates based on nonadiabatic geometric phases. Phys Rev Lett, 2000, 89: 097902[DOI]

4 Berry M V. Quantum phase factor accompanying adiabatic changes. Proc Roy Soc London Ser A, 1984, 392: 45-57[DOI]

5 Aharonov Y, Anandan J. Phase change during a cyclic quantum evolution. Phys Rev Lett, 1987, 58(16): 1593-1596[DOI]

6 Samuel J, Bhandari R. General setting for Berry's phase. Phys Rev Lett, 1988, 60: 2339-2342[DOI]

7 Prado F O, Duzzioni E I, Moussa M H Y, et al. Nonadiabatic coherent evolution of two-level systems under spontaneous decay. Phys Rev Lett, 2009, 102: 073008[DOI]

8 Sjöqvist E, Pati A K, Ekert A, et al. Geometric phases for mixed states in interferometry. Phys Rev Lett, 2000, 85: 2845-2849[DOI]

9 Singh K, Tong D M, Basu K, et al. Geometric phases for nondegenerate and degenerate mixed states. Phys Rev A, 2003, 67: 032106[DOI]

10 Tong D M, Sjoqvist E, Kwek L C, et al. Kinematic approach to the mixed state geometric phase in nonunitary evolution. Phys Rev Lett, 2004, 93: 080405[DOI]

11 Suter D, Mueller K L, Pines A. Study of Aharonov-Anandan quantum phase by NMR interferometer. Phys Rev Lett, 1988, 60: 1218$1220[\mathrm{DOI}$

12 Tian M Z, Reibel R R, Barber Z W, et al. Observation of the geometric phase using photon echoes. Phys Rev A, 2003, 67: 011403[DOI]

13 Tian M Z, Barber Z W, Fischer J A, et al. Geometric manipulation of the quantum states of two-level atoms. Phys Rev A, 2004, 69: $050301(\mathrm{R})$

14 Du J F, Zou P, Shi M G, et al. Observation of geometric phases for mixed states using NMR interferometry. Phys Rev Lett, 2003, 91: 
$100403[\mathrm{DOI}]$

15 Ghosh A, Kumar A. Experimental measurement of mixed state geometric phase by quantum interferometry using NMR. Phys Lett A, 2006, 349: 27-36[DOI]

16 Filipp S, Klepp J, Hasegawa Y, et al. Experimental demonstration of the stability of berry's phase for a spin-1/2 particle. Phys Rev Lett, 2009, 102: 030404[DOI]

17 Wu B, Liu J, Niu Q. Geometric phase for adiabatic evolutions of general quantum states. Phys Rev Lett, 2005, 94: 140402[DOI]

18 曾谨言. 量子力学(II). 北京: 科学出版社, 2001. 224

19 马瑞琼, 李永放, 任立庆, 等. 量子态的相位测量. 中国科学 $\mathrm{G}$ 辑: 物理学 力学 天文学, 2008, 38: 1188-1195

20 马瑞琼, 李永放, 时坚. 相干瞬态的量子干涉效应和 Berry 相位. 物理学报, 2008, 57: 4083-4090

21 李永放, 任立庆, 马瑞琼, 等. 波函数的时域衍射. 中国科学 $\mathrm{G}$ 辑: 物理学 力学 天文学, 2009, 39: 600-605

\title{
Characteristic and measurement of the nonadiabatic and noncyclic evolutive quantum phase
}

\author{
LI YongFang, MA RuiQiong, REN LiQing, WANG Lei, WANG ZhaoHua \& LI BaiHong \\ School of Physics and Information Technology, Shaanxi Normal University, Xi'an 710062, China
}

Based on the model of two-level atoms driven by a time-domain symmetrical light field, the quantum phases of the nonadiabatic and noncyclic evolution are investigated in detail. According to quantum interference effect, a new scheme for the measuring the quantum phases of the non-adiabatic evolution is presented, and the physical mechanism of the measurement quantum phase is expound. The distribution information of the quantum phase can be obtained directly in the population probability of the atomic energy level.

nonadiabatic and noncyclic quantum phase, quantum interference, two-level atomic system 